\title{
Auger recombination in AlGaN quantum wells for UV light-emitting diodes
}

Cite as: Appl. Phys. Lett. 113, 071107 (2018); https://doi.org/10.1063/1.5044383

Submitted: 12 June 2018. Accepted: 01 August 2018 . Published Online: 17 August 2018

Felix Nippert (D), Mohammad Tollabi Mazraehno, Matthew J. Davies, Marc P. Hoffmann, Hans-Jürgen Lugauer (D), Thomas Kure (D), Michael Kneissl, Axel Hoffmann, and Markus R. Wagner (D)

\section{ARTICLES YOU MAY BE INTERESTED IN}

AlGaN-based deep UV LEDs grown on sputtered and high temperature annealed AIN/ sapphire

Applied Physics Letters 112, 041110 (2018); https://doi.org/10.1063/1.5010265

$226 \mathrm{~nm}$ AIGaN/AIN UV LEDs using p-type Si for hole injection and UV reflection Applied Physics Letters 113, 01111 (2018); https://doi.org/10.1063/1.5038044

Enhanced p-type conduction in AIGaN grown by metal-source flow-rate modulation epitaxy Applied Physics Letters 113, 072107 (2018); https://doi.org/10.1063/1.5040334

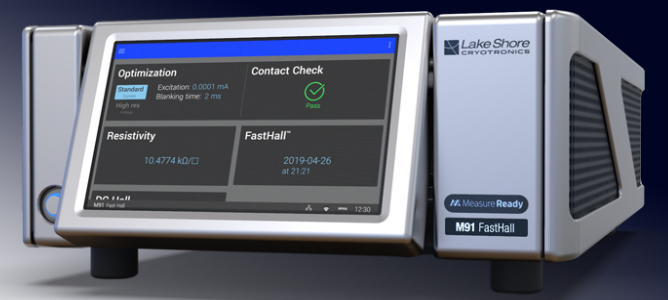

M Measure Ready M91 FastHall ${ }^{\text {TM }}$ Controller

A revolutionary new instrument for complete Hall analysis

See the video 0 


\title{
Auger recombination in AIGaN quantum wells for UV light-emitting diodes
}

\author{
Felix Nippert, ${ }^{1, a)}$ Mohammad Tollabi Mazraehno, ${ }^{1,2}$ Matthew J. Davies, ${ }^{2}$ Marc P. Hoffmann, ${ }^{2}$ \\ Hans-Jürgen Lugauer, ${ }^{2}$ Thomas Kure, ${ }^{1}$ Michael Kneissl, ${ }^{1}$ Axel Hoffmann, ${ }^{1}$ \\ and Markus R. Wagner ${ }^{1}$ \\ ${ }^{1}$ Institut für Festkörperphysik, Technische Universität Berlin, Hardenbergstraße 36, 10623 Berlin, Germany \\ ${ }^{2}$ OSRAM Opto Semiconductors GmbH, Leibnizstraße 4, 93055 Regensburg, Germany
}

(Received 12 June 2018; accepted 1 August 2018; published online 17 August 2018)

\begin{abstract}
We show that the often observed efficiency droop in AlGaN quantum well heterostructures is an internal carrier loss process, analogous to the InGaN system. We attribute this loss process to Auger recombination, with $C=2.3 \times 10^{-30} \mathrm{~cm}^{6} \mathrm{~s}^{-1}$; a similar value found commonly in InGaNbased devices. As a result, the peak internal quantum efficiency (IQE) of our structures is limited to $66 \%$. These values were obtained by resonant excitation (time-resolved) photoluminescence (PL), avoiding common error sources in IQE measurements. The existence of strong Auger recombination implies that simple methods employed for IQE determination, such as temperature-dependent PL, may lead to erroneous values. Auger losses will have to be considered once the challenges regarding carrier injection are solved. Published by AIP Publishing.

https://doi.org/10.1063/1.5044383
\end{abstract}

Ultraviolet (UV) light emitters are required for applications such as disinfection, water purification, UV curing, gas sensing, or optical data storage. In contrast to the visible spectrum, where semiconductor light-emitting diodes (LEDs) have matured into mass-produced commodities, the industrial breakthrough of UV LEDs and laser diodes based on AlGaN quantum wells (QWs) is still hindered by significant technological hurdles. ${ }^{1-3}$ Among other issues, AlGaN-based heterostructures suffer from poor p-type doping ${ }^{4,5}$ and typically show large extraction losses, in particular towards the deep UV spectral range. ${ }^{6,7}$ In comparison to these substantial, external losses, internal losses are usually considered with low priority. Shockley-Read-Hall (SRH) recombination is generally assumed to be dominated by recombination at threading dislocations and therefore to scale only with the threading dislocation density (TDD) ${ }^{8}$ Consequently, low TDDs, as obtained via homoepitaxial growth on bulk-AlN, should lead to internal quantum efficiencies (IQEs) close to unity. The influence of point defects on the IQE is only seldom considered. ${ }^{9}$ Auger recombination, which has been identified as the dominant loss channel at high current densities in InGaN-based LEDs, ${ }^{10-13}$ is generally assumed to be negligible in $\mathrm{AlGaN}$, due to the much larger band gap. We would like to note, however, that the Auger recombination coefficients in InGaN were also found to be much larger than expected, which might be explained by strong coupling to phonons. ${ }^{14}$ Recent studies indirectly suggest that Auger recombination in AlGaN may be of similar magnitude ${ }^{15}$ as compared to InGaN-based devices.

Assessing the IQE of group III-nitride semiconductor devices is non-trivial, as some of the external losses, such as those related to carrier injection, may depend on pump power. In electroluminescence (EL) measurements of AlGaN devices, often similar to the well understood case of InGaN-based emitters, a droop-like behaviour of the quantum efficiency is observed. ${ }^{16-19}$ However, extraction of the IQE through

a)Electronic mail: felix@physik.tu-berlin.de electrically pumped measurements requires reliable modelling of the injection efficiency as a function of current density as well as a good estimation of the extraction efficiency. ${ }^{15}$ Optical pumping measurements are often performed as a means to avoid the necessity of accurate device modelling; however, this poses a separate problem regarding the limited choice of suitable deep UV laser excitation sources. These optical measurements are most frequently performed using readily available ArF-excimer lasers, emitting at $193 \mathrm{~nm}$. The case of ArF excitation is still non-trivial as the light is absorbed in all layers of the heterostructures and the excited carriers need to diffuse and relax into the QWs, potentially recombining (radiatively or non-radiatively) before reaching the QWs. Photoluminescence (PL) measurements on AlGaN heterostructures using an ArF excitation source generally fail to show a saturation of the IQE, as the carrier densities reached in the QW are limited by the transport of carriers e.g., from the quantum barriers to the QWs and/or the absorption in the matrix material before material degradation occurs. Without observation of the efficiency maximum, however, precise determination of the IQE is near impossible. For an IQE estimation based on the temperature dependence of the emission intensity ["high temperature-low temperature method" (HT/LT-M)], one needs to assume an IQE of unity at low temperatures, ${ }^{9}$ which is not necessarily a valid assumption, especially not for all possible excitation power densities. ${ }^{20}$ In the case of power-dependent measurements alone, estimations regarding the active volume are required. Often, these measurements neglect Auger recombination (i.e., use an $\mathrm{AB}$ model) and therefore also lead to misrepresentations of the IQE. ${ }^{21,22}$

Figure 1 displays a calculation of the IQE as a function of peak excited carrier density/carrier generation rate for both ideal pulsed/continuous-wave (CW) optical excitation, in order to demonstrate the differences in the methods of the IQE determination by photoluminescence as described above. In this calculation, the following values are used: $A=1.1 \times 10^{7} \mathrm{~s}^{-1}$, $B=2 \times 10^{-11} \mathrm{~cm}^{3} \mathrm{~s}^{-1}, C=2.3 \times 10^{-30} \mathrm{~cm}^{6} \mathrm{~s}^{-1}$, where $A, B$, and $C$ are the SRH, radiative and Auger recombination 


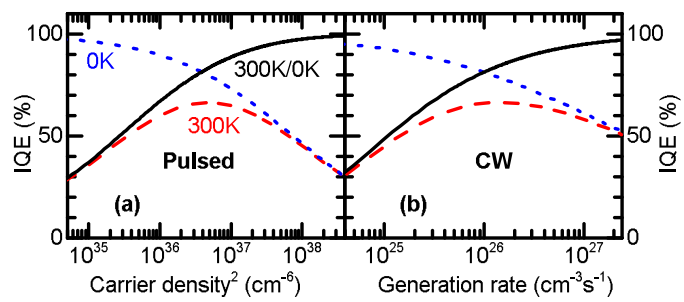

FIG. 1. Power dependent IQE for (a) pulsed or (b) CW excitation. Red dashed lines depict the calculated IQE at room temperature with recombination coefficients as determined in this work (see the text), blue dotted lines use $A=0$ instead, presuming no $\mathrm{SRH}$ recombination at low temperatures. The black lines show the division of room temperature by low temperature results under constant excitation power density, i.e., the inferred IQE from the HT/LT-M measurement, when measured with a single constant excitation power density.

coefficients, respectively. As is commonly assumed,,$^{9,17,22}$ the contribution of SRH recombination is taken as negligible at low temperatures, along with a temperature independent optical absorption coefficient and parameters B and C. In both excitation regimes, the black line represents the result of a fictional measurement using the HT/LT-M. From this calculation, it can be clearly seen that the HT/LT-M IQE tends asymptotically to unity at high excitation powers, if higher order losses are also neglected, and hence would lead to a systematic overestimation of the IQE under both excitation conditions, in the presence of any significant Auger losses. In the case of HT/ LT-M measurements performed at a single excitation power, the resultant IQE can be expected to be even more misleading given that no reference IQE value at low temperatures can be determined. The case of ArF excitation becomes yet more complicated since an accurate determination of the peak carrier density is non-trivial under these conditions. Typical ArF laser pulse durations are between 10 and $30 \mathrm{~ns}$, far from ideal pulsed or ideal $\mathrm{CW}$ conditions. In order to avoid these pitfalls, we investigate AlGaN QWs by means of quasi-resonant PL, allowing us to determine the IQE, as well as the recombination parameters of the $\mathrm{ABC}$ model without many further assumptions.

All samples for this study were grown by metal-organic vapor phase epitaxy on double side polished sapphire substrates using a $3 \mu \mathrm{m}$ thick AlN buffer with a TDD $\approx 10^{9} \mathrm{~cm}^{-2}$. MQWs consisting of $4 \mathrm{~nm}$-thick AIN quantum barriers and $1.5 \mathrm{~nm}$-thick $\mathrm{Al}_{0.45} \mathrm{Ga}_{0.55} \mathrm{~N}$ QWs were grown directly on top of the AlN buffer layer. AlN was selected as a quantum barrier material in order to minimize carrier escape from AlGaN QWs. Four additional samples were also fabricated in order to perform absorption measurements: three MQW samples grown with identical structures and growth conditions except for the number of QWs, namely 5, 15, and 30, and an additional AlN template sample without any MQWs. Finally on top of all structures, a $50 \mathrm{~nm}$-thick AlN cap layer was deposited to avoid any kind of surface effects when characterizing the MQW structures. PL was measured with the third harmonic of a titanium sapphire laser $(247$ to $250 \mathrm{~nm}$ ) with a repetition rate of $80 \mathrm{MHz}$ and a pulse length of about 2 ps. For IQE determination, the luminescent signal was dispersed by a $0.85 \mathrm{~m}$ focal length additive double monochromator (SPEX 1404, 1200 l/mm 500 nm blaze gratings) and recorded with a Princeton Instruments
UV-enhanced charge-coupled device camera. All spectra were acquired with the same integration time (40 s) and constant gain in order to obtain a linear response. For time-resolved measurements (TRPL), the signal was instead recorded through a $0.3 \mathrm{~m}$ focal length Acton SP300i monochromator with a Hamamatsu Ultra Bi-Alkali photo-multiplier tube (H10721-210 cathode) and resolved with time-correlated single photon counting electronics (PicoQuant PicoHarp 300).

We use the $A B C$ rate equation model, ${ }^{23}$ as commonly used to evaluate the IQE of InGaN-based LEDs in a steady state, ${ }^{20,24,25}$ in order to estimate the IQE from our powerdependent PL measurements: The excitation condition is quasi-CW, because the carrier lifetimes and laser pulse separation are of similar magnitude [see low signal dynamic in the TRPL transients in Fig. 3(a)]. Carrier injection losses, which commonly hinder such IQE evaluation in AlGaNbased devices, can be excluded as our excitation is also quasi-resonant, i.e., absorption is expected to occur in the QWs only. We consider SRH losses as they may arise from threading dislocations or point defects and also Auger recombination. The measured (arbitrary units) PL intensity (in counts/s) may be written as $I_{e m}=\eta_{e x t} \eta_{e m} B n^{2}$, where $n$ is the carrier density, taken to be similar for electrons and holes, $V$ is the active volume, $\eta_{\text {ext }}$ is the extraction efficiency, and $\eta_{e m}$ is the efficiency of the detection system for emitted photons. We note that, in general, $n$ is a function of time (due to the pulsed excitation) and space (due to the intensity distribution in the excitation spot) and that $I_{e m}$ is integrated over both space and time. Considering that the emission scales with $n^{2}(r, t)$, whereas the IQE changes very slowly over orders of magnitude of $n(r, t)$, one can assume the IQE to be approximately constant over space and time. The external quantum efficiency is defined as the ratio of photons emitted from the QW to the number of excitation laser photons absorbed. By energy conservation, the latter is equivalent to the total number of recombinations: $\mathrm{EQE}=I_{\text {em }} / I_{\text {exc }}$ $=\eta_{\text {ext }}\left(\eta_{\text {em }} / \eta_{e x}\right) \alpha B n^{2} /\left(A n+B n^{2}+C n^{3}\right)=\eta_{\text {total }}$ IQE, where $\alpha$ is the absorption efficiency, $\eta_{e x}$ is the detection efficiency for the scattered excitation laser and $\eta_{\text {total }}=\eta_{\text {ext }}\left(\eta_{\text {em }} / \eta_{\text {ex }}\right) \alpha$ is a dimensionless factor relating the measured arbitrary units' EQE to the absolute IQE. $I_{e m}$ and $I_{\text {exc }}$ are obtained by integrating over the respective laser and QW emission peaks in the $\mathrm{PL}$ spectra. Maximum $\mathrm{EQE}\left(\mathrm{EQE}_{\text {max }}\right)$ is reached at $n=\sqrt{A / C}$. Normalizing $I_{e m}$ by the intensity $I_{0}=\eta_{e m} \eta_{e x t} B A /$ $C$ at this operating point yields: $\mathrm{EQE}=\eta_{\text {total }} Q /\left(Q+p^{1 / 2}\right.$ $+p^{-1 / 2}$ ), where $Q=B / \sqrt{A C}$ is called the quality factor and $p=I_{e m} / I_{0}$ is the normalized optical output power. It follows that:

$$
\frac{\mathrm{EQE}_{\text {max }}}{\mathrm{EQE}}=\frac{Q+p^{1 / 2}+p^{-1 / 2}}{Q+2}=\frac{\mathrm{IQE}_{\text {max }}}{\mathrm{IQE}},
$$

and

$$
\mathrm{IQE}=\frac{Q}{Q+p^{1 / 2}+p^{-1 / 2}} .
$$

Figure 2(a) shows the integrated QW luminescent signal of the 30-fold MQW structure as a function of input power. 

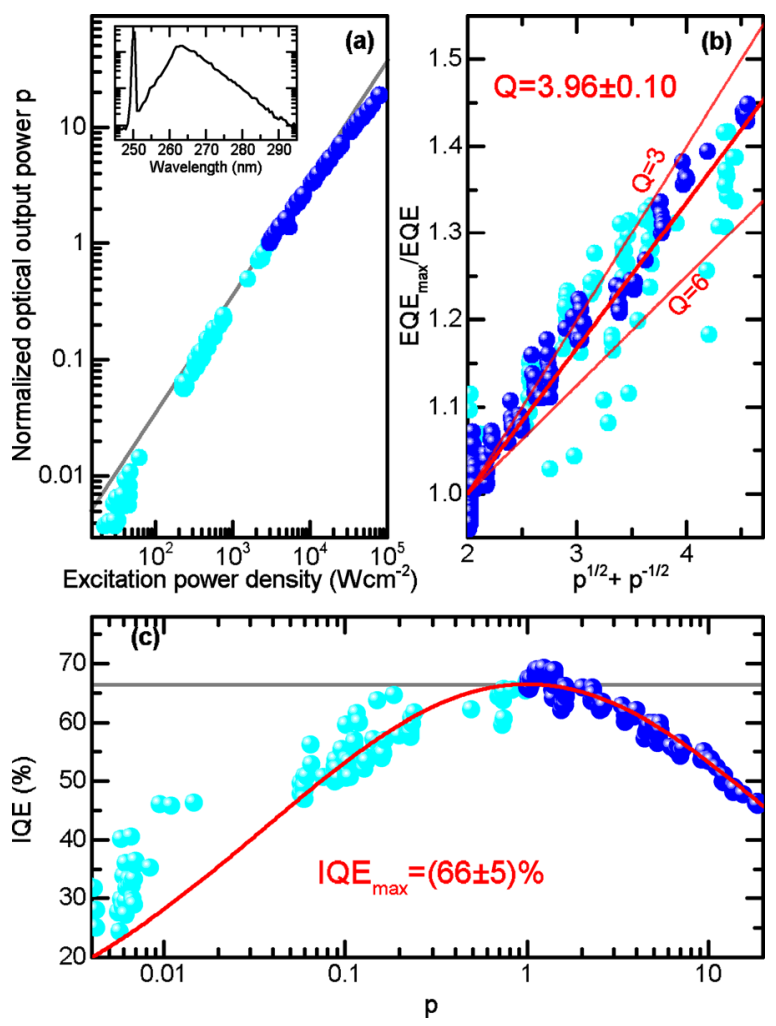

FIG. 2. (a) Normalized optical output power $p$ as a function of excitation power density. For comparison, the grey solid line has a slope of 1 (i.e., constant EQE). Inset: Exemplary PL spectrum with laser and QW emission. (b) Determination of the Quality Factor $Q$ as per Eq. (1). The red line is a fit with $Q=3.96 \pm 0.10$. The thin red lines show $Q=3$ and $Q=6$, respectively, in order to illustrate the robustness of the fit. (c) Estimated IQE as a function of $p$, consistent with $Q$. The red line corresponds to Eq. (2) with $Q=3.96$. In all panels, dark (light) blue datapoints correspond to normalized optical output powers $p>1(p<1)$.

The grey solid line has a slope of one, and would therefore correspond to constant EQE. At high excitation power densities, the emission intensity drops below this line, signifying a non-radiative process growing faster than $n^{2}$. The quasiresonant optical excitation excludes all potential electrical causes (poor carrier injection, low carrier mobility, etc.) as explanations for this behaviour. Instead, the observed nonradiative losses must occur within the QWs. ${ }^{26}$ We suggest that similar observations in EL measurements in comparable structures ${ }^{2,16-19}$ may also be attributed to such internal losses. The shape of the IQE as a function of optical output power [Fig. 2(c)] corresponds to the well-known efficiency droop of InGaN-based LEDs. ${ }^{20,24}$ Indeed, the inclusion of an $n^{3}$ loss, such as Auger recombination in the model, allows for a satisfactory fitting of the IQE [red line in Figs. 2(b) and 2(c)]. At room temperature, we observe $Q=4$ (see thin red lines for a rough estimation of the error interval), corresponding to a maximum IQE ( $\left.\mathrm{IQE}_{\text {max }}\right)$ of $(66 \pm 5) \%$. We note that the deviation towards higher IQE at very low pump powers may be associated with excitonic effects. In general, however, free carriers appear to dominate the optical properties and no signatures of excitonic emission are observed.

In order to estimate the values of the individual recombination coefficients, further measurements are required. Generally, a differential carrier lifetime measurement would be preferable, ${ }^{25}$ but could not be performed here, because the required laser powers for a pump-probe experiment were not available. Instead, we performed standard time-resolved PL, as shown in Fig. 3(a). The PL intensity as a function of delay time is given by $I_{e m}(t)=\eta_{e m} \eta_{e x t} B n(t)^{2}$. Using the relationship $p(t)=n(t)^{2} C / A$, one may define $1 / \tau(t)=\left(d I_{e m}(t) / d t\right) /$ $I_{e m}(0)=(d n(t) / d t) / n(0)$, giving the effective carrier lifetime

$$
\frac{1}{\tau}=2 A(1+Q \sqrt{p}+p)
$$

Figure 3(b) shows the extracted initial carrier lifetimes (exponential decay within the first ns) $\tau$ as a function of $p$, as well as the IQE at these operating points. The green line shows a fit using Eq. (3), yielding a value of around $A=1.1 \times 10^{7} \mathrm{~s}^{-1}$ for the SRH recombination coefficient. The green shaded area shows the expected initial lifetimes for a
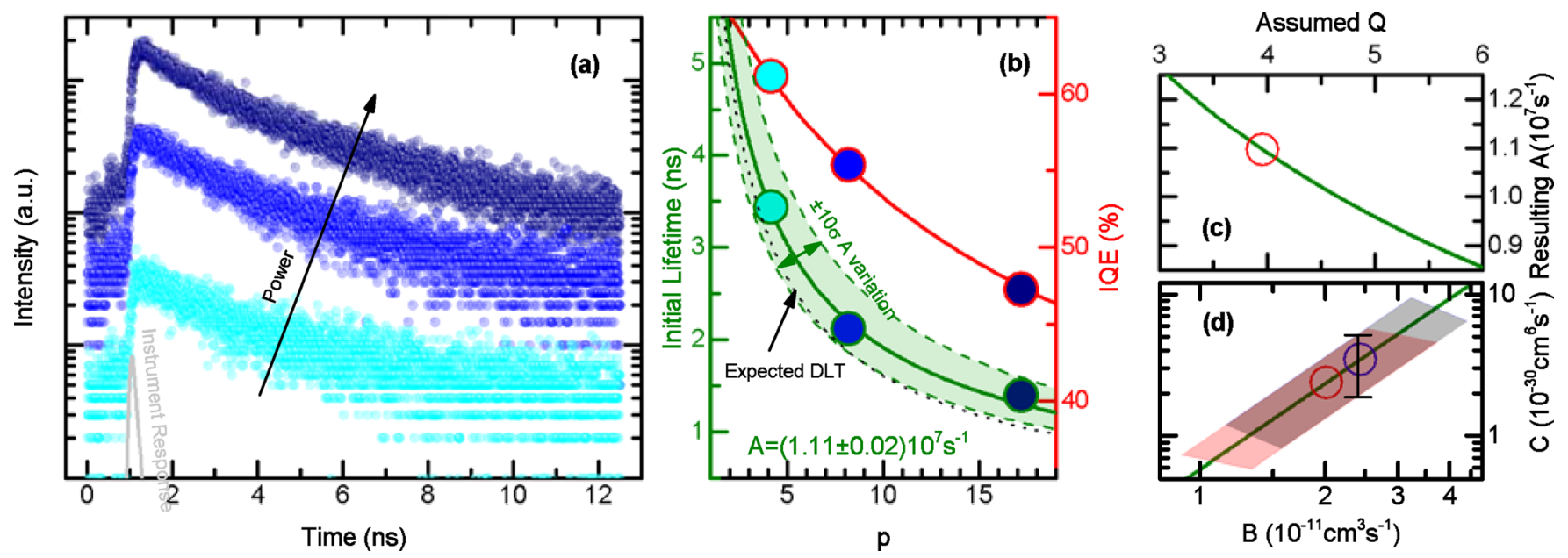

FIG. 3. (a) Time-resolved PL at three different excitation powers (offset for clarity). (b) IQE at the corresponding three operating points (red) and correlation to Fig. 2(c). Initial carrier lifetime [green, from the slope of decay within the first ns in (a)] and fit to Eq. (3). Green dashed lines: Variation of $A$ by $10 \sigma$. Black dotted line: Corresponding expected differential carrier lifetime. ${ }^{25}$ (c) Variation of the estimated value for $A$ as a function of $Q$ in the range of values shows by the thin lines in Fig. 2(b). The red circle marks the values found by fitting. (d) $C$ as a function of $B$ as implied by $B^{2} / C=Q^{2} A$. The black bar marks an oftenused value for $B$ from the literature, ${ }^{27}$ along with the implied $C$ (uncertainty in $B^{2} / C$, as determined here). The red (blue) circle shows our estimation of the coefficients assuming CW (ideal pulsed) excitation conditions (see main text). The shaded areas reflect the error estimation. The main source of error is uncertainty in active volume. 
variation of $\pm 10 \sigma$, indicating the robustness of the fit. We note that the extracted value of $A$ is more sensitive to variation in $Q$ [see Fig. 3(c), compare thin lines in Fig. 2(b)]. We therefore estimate $A=(1.1 \pm 0.2) \times 10^{7} \mathrm{~s}^{-1}$. The extracted values for $Q$ and $A$ also imply $B^{2} C^{-1}=1.74 \times 10^{8} \mathrm{~s}^{-1}$ [see Fig. 3(d)]. The other two recombination coefficients follow from the relations $B=A^{2} Q(Q+2) / j, C=A^{3}(Q+2)^{2} / j^{2}$, where $j$ is the injection rate per unit volume at peak efficiency, ${ }^{25}$ which depends on input power density per unit area, absorption of the $\mathrm{QW}$ at a given wavelength and QW thickness. In order to make an accurate determination of the parameters $B$ and $C$, we have performed transmission and reflection measurements on the series of equivalent MQW structures with increasing numbers of QWs described above, along with the bulk template structure. The determined optical absorption is $(0.57 \pm 0.02) \%$ per QW at $250 \mathrm{~nm}$. The spot area was determined to be $18 \mu \mathrm{m}^{2}$, giving $j=1.4 \times 10^{26} \mathrm{~cm}^{-3} \mathrm{~s}^{-1}$, which corresponds to a current density per QW of $3.5 \mathrm{~A} \mathrm{~cm}^{-2}$. The radiative (Auger) recombination coefficient is therefore $2 \times 10^{-11} \mathrm{~cm}^{3} \mathrm{~s}^{-1}\left(2.3 \times 10^{-30} \mathrm{~cm}^{6} \mathrm{~s}^{-1}\right)$, see the red circle in Fig. 3(d), where the red shaded area shows an estimation of the uncertainty range as caused by uncertainty in the spot size, the QW thickness and $Q$. While the measurement conditions can be considered to be close to $\mathrm{CW}$ conditions, one should keep in mind that the ABC model is only strictly valid in the steady-state condition. Hence, for comparison, we have computed the recombination coefficients under the assumption that the carrier density is governed by the pulse energy only (i.e., ideal pulsed conditions). In this case, the initial carrier density at peak efficiency is $n_{0}=1.8 \times 10^{18} \mathrm{~cm}^{-3}$ and we obtain $B=2.4 \times 10^{-11} \mathrm{~cm}^{3} \mathrm{~s}^{-1}$ and $C=3.4 \times 10^{-30}$ $\mathrm{cm}^{6} \mathrm{~s}^{-1}$, respectively [blue circle and shaded area in Fig. $3(d)]$. We estimate the effective density of states in the conduction band to be around $2 \times 10^{19} \mathrm{~cm}^{-3}$ at room temperature. The maximum inferred carrier density reached during our experiment at $p \approx 20$ is around $8 \times 10^{18} \mathrm{~cm}^{-3}$, and therefore not large enough to introduce significant carrier degeneracy effects.

We note that a similar value for $B$ has been found experimentally in $\mathrm{GaN},{ }^{27}$ which is often assumed to be valid for AlGaN as well ${ }^{15,21,22}$ [black bar in Fig. 3(d)], whereas no reliable literature data exist on $C$. We also emphasize that the Auger recombination coefficient determined here is very similar to values found in $\mathrm{InGaN}$-based devices. ${ }^{20,25,28}$ In both cases, $C$ is much higher than traditionally expected for direct semiconductors with such large band gaps. Furthermore, a significant reduction of SRH recombination, for example, by two orders of magnitude, would imply an increase in $\mathrm{IQE}_{\max }$ to $95 \%$ for our structure. Such a reduction could be obtained on AlN substrates, unless $A$ is limited by point defects. We would like to highlight that while different types of simple IQE measurements can be helpful for comparing sets of similar samples (e.g., growth parameter series), based on the previously discussed assumptions, the extracted number is unlikely representative of the true IQE. The method presented here allows for accurate IQE determination provided that the excitation power density is sufficient to surpass the maximum of IQE, without any assumptions on the active volume or absolute excitation power density, which only need to be determined for the parameters $B$ and $C$.

In conclusion, we have shown that efficiency droop in AlGaN QWs is a QW internal loss mechanism, which behaves near identical to the well-known case of InGaN/ GaN QWs. We attribute the reduction in IQE at high excitation power density to the losses associated with Auger recombination and have extracted an Auger recombination coefficient of $C=2.3 \times 10^{-30} \mathrm{~cm}^{6} \mathrm{~s}^{-1}$, which is of the same order as found in $\mathrm{InGaN} / \mathrm{GaN}$ QWs. We have demonstrated that proper estimation of the IQE requires resonant excitation and pumping beyond the maximum of efficiency, along with the importance of considering the losses due to Auger recombination in determining the IQE.

We gratefully acknowledge the financial support of the German Science Foundation within the Collaborative Research Center 787 (CRC 787).

${ }^{1}$ M. Kneissl, T. Kolbe, C. Chua, V. Kueller, N. Lobo, J. Stellmach, A. Knauer, H. Rodriguez, S. Einfeldt, Z. Yang, N. M. Johnson, and M. Weyers, Semicond. Sci. Technol. 26, 014036 (2011).

${ }^{2}$ H. Hirayama, N. Maeda, S. Fujikawa, S. Toyoda, and N. Kamata, Jpn. J. Appl. Phys., Part 1 53, 100209 (2014).

${ }^{3}$ J.-S. Park, J. K. Kim, J. Cho, and T.-Y. Seong, ECS J. Solid State Sci. Technol. 6, Q42 (2017).

${ }^{4}$ C. G. Van de Walle, C. Stampfl, and J. Neugebauer, J. Cryst. Growth 189-190, 505 (1998).

${ }^{5}$ K. B. Nam, M. L. Nakarmi, J. Li, J. Y. Lin, and H. X. Jiang, Appl. Phys. Lett. 83, 878 (2003).

${ }^{6}$ T. Kolbe, A. Knauer, C. Chua, Z. Yang, S. Einfeldt, P. Vogt, N. M. Johnson, M. Weyers, and M. Kneissl, Appl. Phys. Lett. 97, 171105 (2010).

${ }^{7}$ C. Reich, M. Guttmann, M. Feneberg, T. Wernicke, F. Mehnke, C. Kuhn, J. Rass, M. Lapeyrade, S. Einfeldt, A. Knauer, V. Kueller, M. Weyers, R. Goldhahn, and M. Kneissl, Appl. Phys. Lett. 107, 142101 (2015).

${ }^{8}$ S. Y. Karpov and Y. N. Makarov, Appl. Phys. Lett. 81, 4721 (2002).

${ }^{9}$ M. Shatalov, W. Sun, A. Lunev, X. Hu, A. Dobrinsky, Y. Bilenko, J. Yang, M. Shur, R. Gaska, C. Moe, G. Garrett, and M. Wraback, Appl. Phys. Express 5, 082101 (2012).

${ }^{10}$ J. Iveland, L. Martinelli, J. Peretti, J. S. Speck, and C. Weisbuch, Phys. Rev. Lett. 110, 177406 (2013).

${ }^{11}$ B. Galler, H.-J. Lugauer, M. Binder, R. Hollweck, Y. Folwill, A. Nirschl, A. Gomez-Iglesias, B. Hahn, J. Wagner, and M. Sabathil, Appl. Phys. Express 6, 112101 (2013).

${ }^{12}$ M. Binder, A. Nirschl, R. Zeisel, T. Hager, H.-J. Lugauer, M. Sabathil, D. Bougeard, J. Wagner, and B. Galler, Appl. Phys. Lett. 103, 071108 (2013).

${ }^{13}$ E. Kioupakis, Q. Yan, D. Steiauf, and C. G. Van de Walle, New J. Phys. 15, 125006 (2013).

${ }^{14}$ E. Kioupakis, P. Rinke, K. T. Delaney, and C. G. Van de Walle, Appl. Phys. Lett. 98, 161107 (2011).

${ }^{15}$ G.-D. Hao, N. Tamari, T. Obata, T. Kinoshita, and S. Inoue, Opt. Express 25, A639 (2017).

${ }^{16}$ W. Sun, M. Shatalov, J. Deng, X. Hu, J. Yang, A. Lunev, Y. Bilenko, M. Shur, and R. Gaska, Appl. Phys. Lett. 96, 061102 (2010).

${ }^{17}$ J. Mickevicius, G. Tamulaitis, M. Shur, M. Shatalov, J. Yang, and R. Gaska, Appl. Phys. Lett. 103, 011906 (2013).

${ }^{18}$ M. Shatalov, W. Sun, R. Jain, A. Lunev, X. Hu, A. Dobrinsky, Y. Bilenko, J. Yang, G. A. Garrett, L. E. Rodak, M. Wraback, M. Shur, and R. Gaska, Semicond. Sci. Technol. 29, 084007 (2014).

${ }^{19}$ J. Yun, J.-I. Shim, and H. Hirayama, Appl. Phys. Express 8, 022104 (2015). 
${ }^{20}$ F. Nippert, S. Y. Karpov, G. Callsen, B. Galler, T. Kure, C. Nenstiel, M. R. Wagner, M. Straßburg, H.-J. Lugauer, and A. Hoffmann, Appl. Phys. Lett. 109, 161103 (2016).

${ }^{21}$ K. Ban, J. Yamamoto, K. Takeda, K. Ide, M. Iwaya, T. Takeuchi, S. Kamiyama, I. Akasaki, and H. Amano, Appl. Phys. Express 4, 052101 (2011).

${ }^{22}$ Z. Bryan, I. Bryan, J. Xie, S. Mita, Z. Sitar, and R. Collazo, Appl. Phys. Lett. 106, 142107 (2015).

${ }^{23}$ S. Karpov, Opt. Quantum Electron. 47, 1293 (2015).

${ }^{24}$ I. Titkov, S. Karpov, A. Yadav, V. Zerova, M. Zulonas, B. Galler, M. Strassburg, I. Pietzonka, H.-J. Lugauer, and E. Rafailov, IEEE J. Quantum Electron. 50, 911 (2014).
${ }^{25}$ F. Nippert, S. Karpov, I. Pietzonka, B. Galler, A. Wilm, T. Kure, C. Nenstiel, G. Callsen, M. Straßburg, H.-J. Lugauer, and A. Hoffmann, Jpn. J. Appl. Phys., Part 1 55, 05FJ01 (2016).

${ }^{26}$ A. Laubsch, M. Sabathil, W. Bergbauer, M. Strassburg, H. Lugauer, M. Peter, S. Lutgen, N. Linder, K. Streubel, J. Hader, J. V. Moloney, B. Pasenow, and S. W. Koch, Phys. Status Solidi (c) 6, S913 (2009).

${ }^{27}$ J. S. Im, A. Moritz, F. Steuber, F. Scholz, and A. Hangleiter, Appl. Phys. Lett. 70, 631 (1997).

${ }^{28}$ B. Galler, P. Drechsel, R. Monnard, P. Rode, P. Stauss, S. Froehlich, W. Bergbauer, M. Binder, M. Sabathil, B. Hahn, and J. Wagner, Appl. Phys. Lett. 101, 131111 (2012). 